\title{
Supplementary Materials for Analysis of miRNA-mRNA crosstalk in Radiation-Induced Mouse Thymic Lymphomas to identify miR-486 as a critical regulator by targeting IGF2BP3 mRNA
}

Hainan Zhaob\#, Suhe Donga, Jicong Dü\#, Penglin Xia ${ }^{\mathrm{a}}$, Ruling Liu ${ }^{\mathrm{a}}$, Tingting Liu ${ }^{\mathrm{a}}$, Yajie Yang ${ }^{\mathrm{c}}$, Ying Cheng ${ }^{\mathrm{a}}$, Jianming $\mathrm{Cai}^{\mathrm{a}}$, Cong $\mathrm{Liu}^{\mathrm{a} *}, \mathrm{Fu} \mathrm{Gao}^{\mathrm{a} *}, \mathrm{Hu} \mathrm{Liu}^{\mathrm{a} *}$

\# Hainan Zhao, Suhe Dong, Jicong Du contributed equally to this work

*Corresponding author: Hu Liu, Email: gzsassliuhu@163.com; Fu Gao, Email: gaofusmmu@163.com; Cong Liu,Email: victorliu20102020@163.com

This PDF file includes:

Fig. S1. mRNAs expression in RTL tissues with GO classification and KEGG pathways analysis.

Fig. S2. 3 of the most important signaling pathways involved in the process of RTL.

Tab. S1. All differentially expressed mouse miRNAs in comparisons of T1-T3 VS C1-C3

Tab. S2. Part of differentially expressed mouse genes in comparisons of T1-T3 VS C1-C3
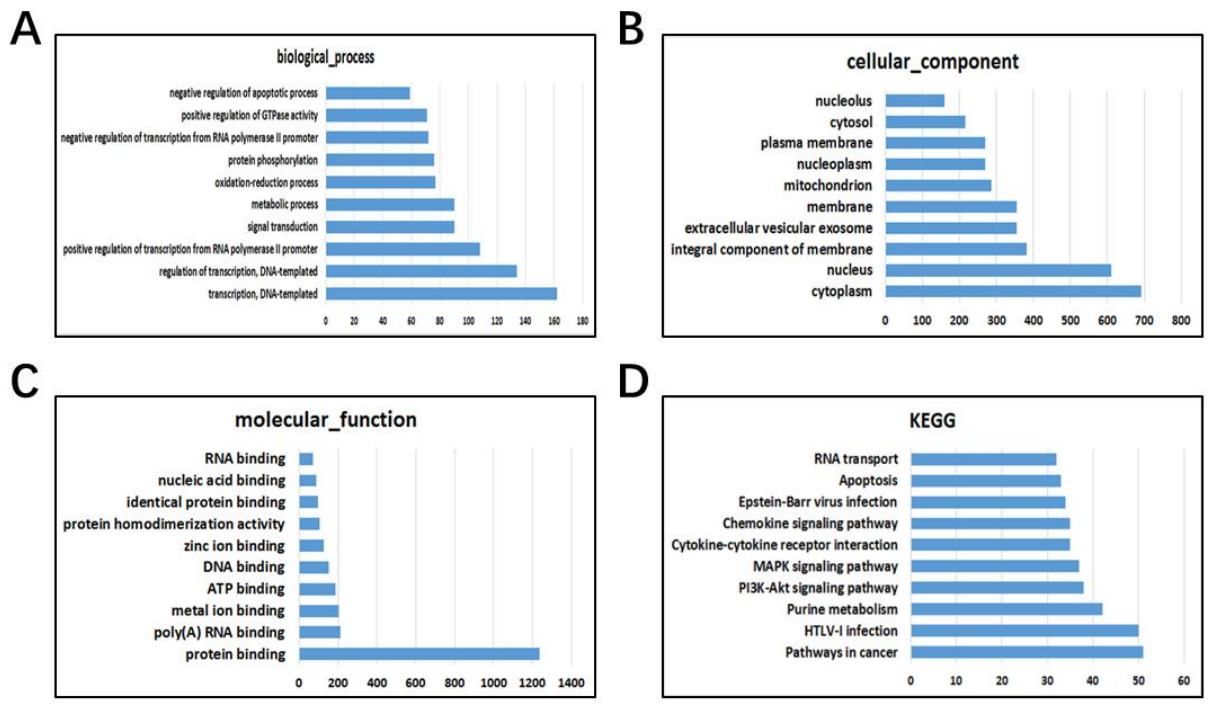

Fig S1. GO Classification and KEGG Pathways analysis of mRNAs: The functional annotation of mRNAs between RTL tissues and normal thymus tissues was conducted by GO Classification and KEGG Pathways analysis. The differentially expressed mRNAs were enriched into biological processes (A), cellular component (B) and molecular functions (C). (D) shows 10 of the most important signaling pathways in which the cancer signaling pathway is most prominent in the RTL organization, which is also very practical. 


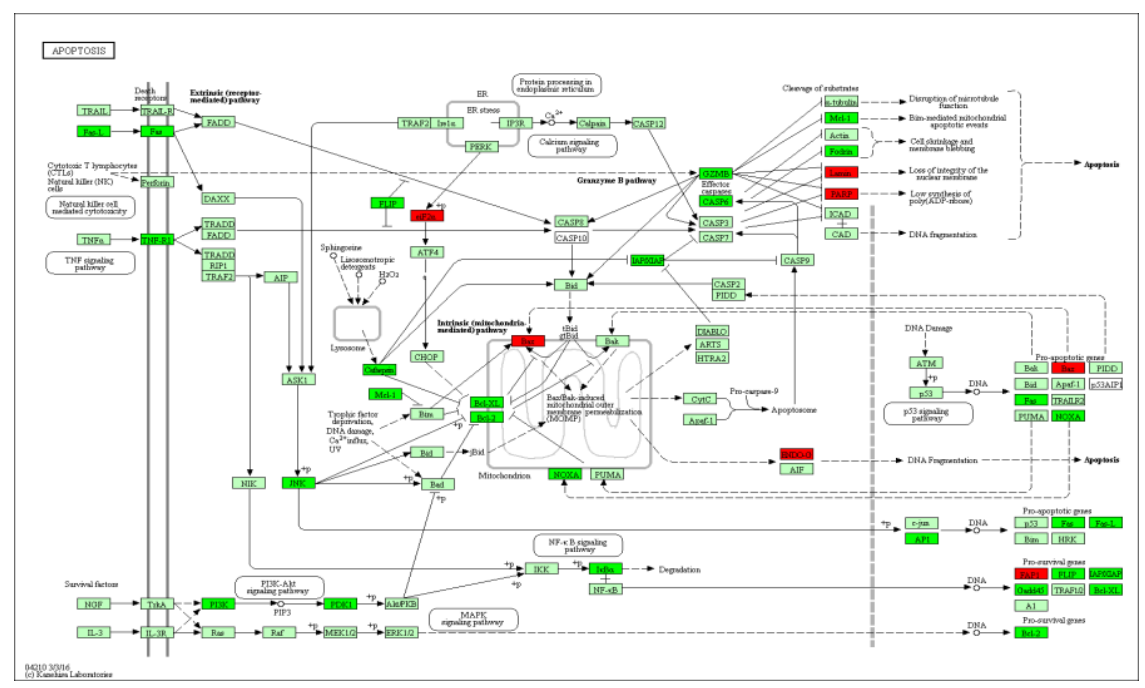

Fig S2-A Apoptosis pathway

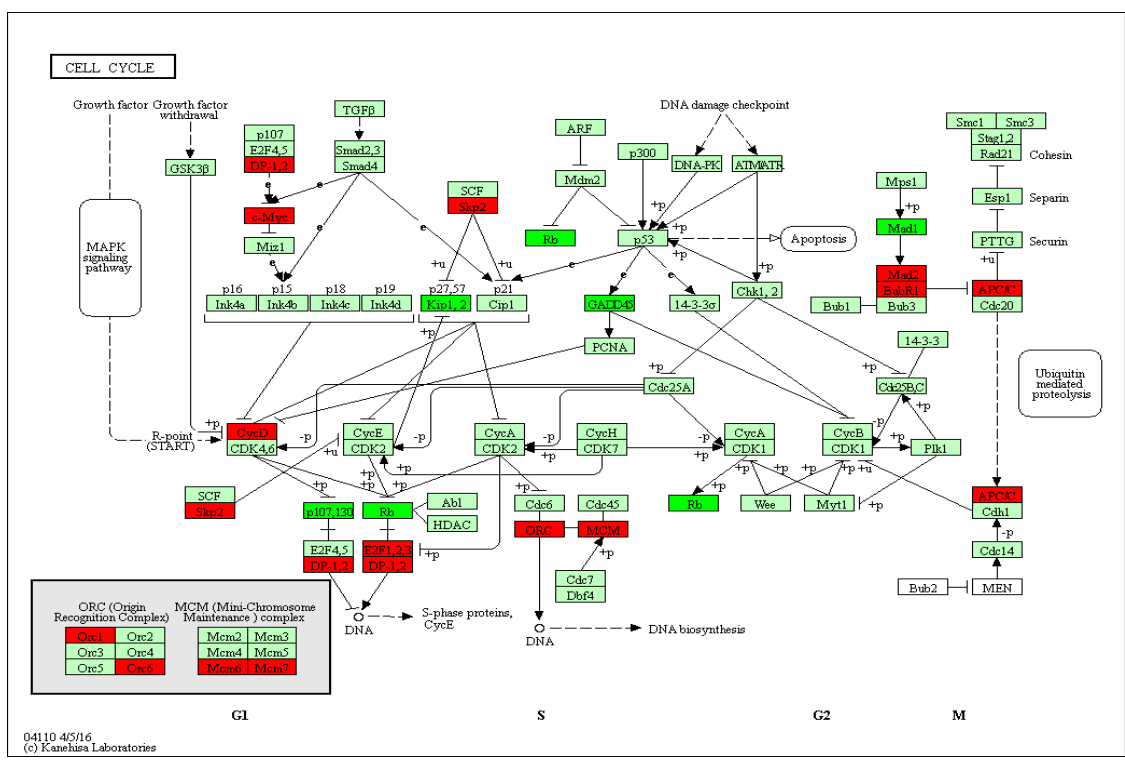

Fig S2-B Cell cycle pathway

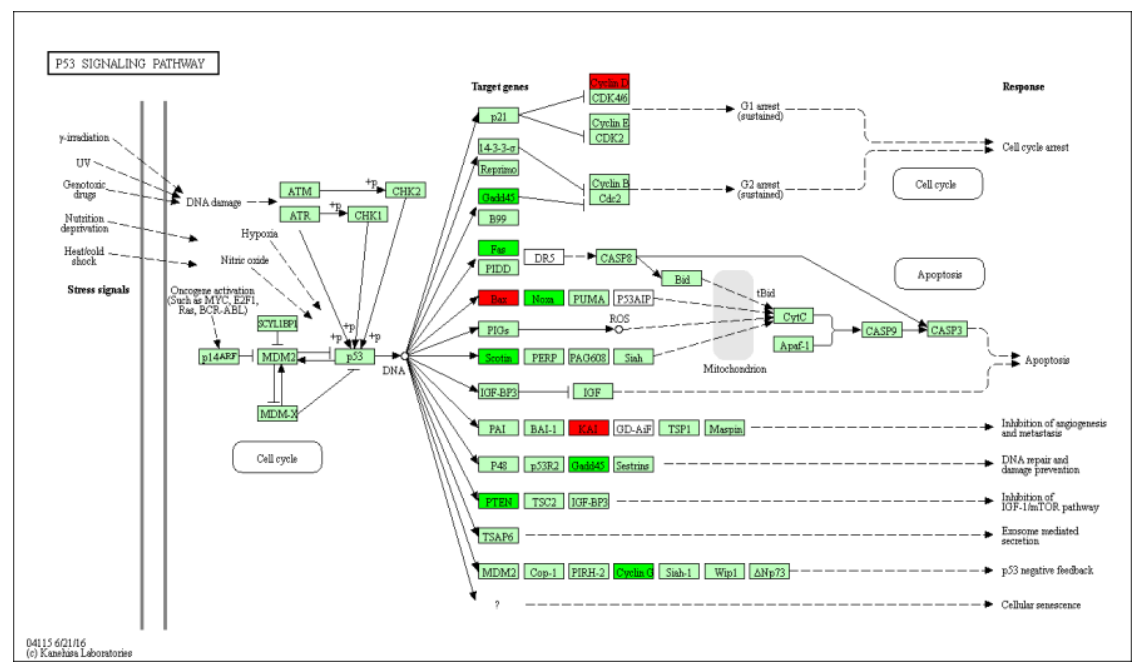

Fig S2-C Cell cycle pathway 
Tab S1. All differentially expressed mouse miRNAs in comparisons of

T1-T3 VS C1-C3

\begin{tabular}{|c|c|c|c|c|c|c|c|}
\hline miRNA & $\begin{array}{c}\text { Fold } \\
\text { Change }\end{array}$ & $\mathrm{C} 1$ & $\mathrm{C} 2$ & $\mathrm{C3}$ & T1 & $\mathbf{T} 2$ & T3 \\
\hline mmu-miR-762 & 16.29995 & 53.1785 & 79.0491 & 56.7184 & 830.121 & 1054.67 & 1195.01 \\
\hline mmu-miR-1224 & 15.05824 & 14.6422 & 28.3890 & 19.3253 & 144.937 & 389.691 & 404.353 \\
\hline mmu-miR-714 & 11.84064 & 25.2979 & 20.3164 & 10.0204 & 205.086 & 204.814 & 248.85 \\
\hline mmu-miR-467a & 8.993497 & 39.6074 & 22.3869 & 20.2478 & 236.720 & 205.876 & 297.048 \\
\hline mmu-miR-455 & 8.088613 & 31.2109 & 32.9377 & 25.3580 & 123.804 & 398.679 & 201.501 \\
\hline mmu-miR-699 & 7.029267 & 17.4616 & 15.2747 & 24.4927 & 118.171 & 143.404 & 140.703 \\
\hline mmu-miR-712 & 6.428803 & 18.3208 & 12.0471 & 17.2920 & 89.4958 & 104.471 & 112.429 \\
\hline mmu-miR-685 & 6.162833 & 88.6207 & 38.1896 & 119.005 & 432.877 & 601.174 & 480.871 \\
\hline mmu-miR-705 & 6.105099 & 39.0689 & 38.0218 & 20.8677 & 135.672 & 226.754 & 235.619 \\
\hline mmu-miR-689 & 5.986064 & 11.6245 & 19.6520 & 19.2770 & 103.993 & 80.2785 & 118.345 \\
\hline mmu-miR-494 & 5.65435 & 34.4538 & 49.4370 & 20.3338 & 308.007 & 151.878 & 129.436 \\
\hline mmu-miR-711 & 4.928502 & 16.6324 & 13.1007 & 15.21 & 69.6823 & 67.1815 & 84.6387 \\
\hline mmu-miR-181d & 4.048975 & 83.2381 & 103.739 & 37.3784 & 361.327 & 294.601 & 252.483 \\
\hline mmu-miR-149 & 3.740021 & 44.2006 & 43.2228 & 37.4694 & 159.746 & 231.614 & 75.7407 \\
\hline mmu-miR-466c-5p & 3.560261 & 25.4234 & 22.4260 & 18.7778 & 81.9984 & 84.2913 & 70.9211 \\
\hline mmu-miR-720 & 3.39201 & 141.401 & 148.214 & 134.330 & 462.927 & 446.525 & 528.578 \\
\hline mmu-miR-744 & 3.061094 & 18.2514 & 24.1779 & 17.7233 & 62.7648 & 46.3654 & 75.0026 \\
\hline mmu-miR-210 & 3.054034 & 85.1111 & 145.708 & 117.247 & 167.083 & 658.138 & 237.785 \\
\hline mmu-miR-669c & 3.03839 & 25.5928 & 40.6563 & 12.7917 & 95.2017 & 68.0033 & 76.9521 \\
\hline mmu-miR-671-5p & 2.939634 & 18.9173 & 11.1667 & 13.6123 & 26.1858 & 56.3387 & 45.9271 \\
\hline mmu-miR-466b & 2.840524 & 14.3840 & 12.5811 & 20.5821 & 43.6671 & 51.8091 & 39.5830 \\
\hline mmu-miR-93 & 2.759742 & 20.7872 & 31.2408 & 23.6820 & 75.2095 & 75.2292 & 58.5013 \\
\hline mmu-miR-130b & 2.71546 & 341.970 & 325.832 & 359.930 & 638.742 & 1072.91 & 1079.11 \\
\hline mmu-miR-21 & 2.711959 & 45.8795 & 38.8022 & 15.8378 & 58.5999 & 93.9895 & 120.015 \\
\hline mmu-miR-345-5p & 2.616764 & 29.9252 & 19.4661 & 59.4024 & 82.6259 & 124.592 & 77.4696 \\
\hline mmu-miR-28-star & 2.608482 & 23.2207 & 14.1079 & 22.9167 & 44.647 & 58.1214 & 54.3806 \\
\hline mmu-miR-324-5p & 2.59365 & 40.3607 & 46.3655 & 19.2582 & 88.0344 & 98.6218 & 88.2304 \\
\hline mmu-miR-466a-3p & 2.584312 & 16.2989 & 17.1604 & 10.2015 & 43.8735 & 26.3014 & 42.6582 \\
\hline mmu-miR-181c & 2.553082 & 56.5037 & 55.5920 & 68.6313 & 115.924 & 208.044 & 137.442 \\
\hline mmu-miR-181b & 2.510714 & 1697.41 & 952.985 & 860.377 & 3528.40 & 3001.48 & 2284.65 \\
\hline
\end{tabular}




\begin{tabular}{|c|c|c|c|c|c|c|c|}
\hline mmu-miR-467c & 2.371236 & 17.7430 & 16.7794 & 14.8608 & 39.3922 & 22.7937 & 54.9135 \\
\hline mmu-miR-345-3p & 2.366719 & 14.0309 & 17.4256 & 11.4777 & 31.7484 & 33.6383 & 36.2266 \\
\hline mmu-miR-466e-3p & 2.342553 & 22.1523 & 19.5949 & 15.4438 & 59.0403 & 33.9539 & 40.9791 \\
\hline mmu-miR-192 & 2.337433 & 25.5656 & 18.5268 & 13.8254 & 43.5146 & 56.3697 & 35.4949 \\
\hline mmu-miR-106b & 2.335864 & 457.508 & 382.235 & 496.206 & 950.837 & 1208.90 & 960.854 \\
\hline mmu-miR-1198 & 2.316914 & 27.6248 & 20.5274 & 40.0138 & 70.7933 & 62.7140 & 70.7661 \\
\hline mmu-miR-106a & 2.188859 & 675.125 & 563.677 & 641.697 & 1415.55 & 1457.60 & 1242.99 \\
\hline mmu-miR-106b & 2.184321 & 102.695 & 98.5000 & 111.266 & 167.298 & 303.598 & 211.620 \\
\hline mmu-miR-669a & 2.171295 & 16.2217 & 27.3903 & 19.9955 & 48.6795 & 40.9565 & 48.4748 \\
\hline mmu-miR-297a & 2.126719 & 25.1735 & 26.7657 & 21.7732 & 46.4573 & 54.7988 & 55.5097 \\
\hline mmu-miR-669d & 2.105619 & 14.9034 & 9.94151 & 14.6326 & 23.0809 & 35.0980 & 24.9458 \\
\hline mmu-miR-30c & 2.038946 & 14.1193 & 12.242 & 12.0391 & 29.9951 & 25.7418 & 22.5595 \\
\hline mmu-miR-421 & 2.028943 & 22.8130 & 21.2772 & 16.3328 & 50.6702 & 30.8309 & 41.0939 \\
\hline mmu-miR-423-3p & 2.022929 & 112.956 & 85.5128 & 74.5041 & 182.305 & 216.634 & 153.267 \\
\hline mmu-miR-20b & 2.014202 & 832.639 & 804.973 & 775.421 & 1735.19 & 1877.51 & 1247.63 \\
\hline mmu-miR-20b & 2.013521 & 13.6491 & 12.3863 & 20.0198 & 31.8858 & 43.0954 & 17.7520 \\
\hline mmu-miR-320 & 0.470245 & 162.356 & 167.129 & 129.259 & 63.3613 & 81.4806 & 70.8803 \\
\hline mmu-let-7e & 0.456293 & 371.518 & 512.564 & 498.025 & 152.590 & 186.752 & 291.304 \\
\hline mmu-miR-31 & 0.438599 & 91.2414 & 69.5214 & 89.6808 & 31.8596 & 38.3885 & 39.5963 \\
\hline mmu-miR-150 & 0.42951 & 489.47 & 438.772 & 436.658 & 277.347 & 134.451 & 174.443 \\
\hline mmu-miR-145 & 0.423382 & 690.768 & 992.685 & 944.353 & 316.297 & 374.508 & 421.759 \\
\hline mmu-miR-125a-5p & 0.397347 & 211.183 & 253.218 & 217.857 & 74.6584 & 62.1876 & 134.247 \\
\hline mmu-miR-376b & 0.367487 & 216.377 & 383.566 & 439.559 & 101.424 & 154.294 & 126.284 \\
\hline mmu-miR-708 & 0.363598 & 60.0487 & 69.8726 & 73.9306 & 23.0969 & 18.7564 & 32.2668 \\
\hline mmu-miR-152 & 0.352634 & 91.5288 & 158.328 & 124.199 & 29.7740 & 40.9162 & 61.2146 \\
\hline mmu-miR-143 & 0.309535 & 636.801 & 688.172 & 629.305 & 204.821 & 192.416 & 207.679 \\
\hline mmu-miR-200c & 0.290216 & 359.078 & 405.968 & 344.590 & 147.720 & 48.5455 & 125.768 \\
\hline mmu-miR-193b & 0.265919 & 113.374 & 167.355 & 153.267 & 46.4803 & 27.1019 & 41.8259 \\
\hline mmu-miR-203 & 0.263256 & 86.3613 & 46.1323 & 48.4723 & 15.2790 & 16.0102 & 16.3511 \\
\hline mmu-miR-100 & 0.231036 & 93.5010 & 128.056 & 111.354 & 31.7794 & 17.5401 & 27.5951 \\
\hline mmu-miR-99a & 0.224574 & 207.403 & 302.896 & 204.287 & 56.6218 & 57.3419 & 46.5137 \\
\hline mmu-miR-486 & 0.172003 & 1225.26 & 1454.16 & 1017.27 & 256.023 & 293.051 & 86.7695 \\
\hline mmu-miR-125b & 0.154761 & 1718.73 & 1794.66 & 1692.97 & 280.492 & 231.610 & 293.642 \\
\hline
\end{tabular}

Red mark indicates up-regulated miRNAs in RTL tissues and Green mark indicates down-regulation. "C" represents control group that is normal normal thymus tissues; "T" represents RTL tissues. 
Tab S2. Part of differentially expressed mouse genes in comparisons of T1-T3 VS C1-C3

\begin{tabular}{|c|c|c|c|c|c|c|c|}
\hline Gene Symbol & $\begin{array}{c}\text { Fold } \\
\text { Change }\end{array}$ & T1 & $\mathbf{T 2}$ & T3 & C1 & C2 & C3 \\
\hline Gm266 & 260.4477 & 4271.2417 & 5640.1011 & 912.71798 & 12.715723 & 9.7900993 & 9.9974218 \\
\hline Fbp1 & 61.8923 & 1207.7365 & 4856.5568 & 2172.1313 & 45.482054 & 44.898985 & 26.314815 \\
\hline Pdgfrb & 51.8299 & 983.55009 & 4055.8402 & 1914.1685 & 31.320948 & 40.883162 & 42.828917 \\
\hline Col1a2 & 33.9661 & 88.411114 & 2005.2151 & 2913.9344 & 17.161091 & 32.222048 & 23.84027 \\
\hline Trim2 & 28.4234 & 407.53067 & 1198.0053 & 175.15596 & 15.346666 & 16.419607 & 14.778726 \\
\hline Spsb4 & 28.0073 & 800.37678 & 973.4989 & 442.76773 & 23.23589 & 22.584869 & 29.923645 \\
\hline Ripk4 & 27.2514 & 1369.1929 & 203.06926 & 706.35599 & 19.005196 & 22.002647 & 23.207107 \\
\hline EG667874 & 26.2544 & 1125.9344 & 908.65898 & 417.49604 & 25.681892 & 31.239385 & 29.4191 \\
\hline Ddc & 26.0797 & 1126.7894 & 917.93561 & 1430.0368 & 39.827739 & 39.792378 & 52.614759 \\
\hline Trim2 & 22.1869 & 636.38209 & 1720.7716 & 360.07674 & 24.835553 & 43.333677 & 33.54632 \\
\hline Emx2 & 21.7221 & 69.509618 & 434.04012 & 242.45878 & 8.6898175 & 9.4512199 & 8.6898175 \\
\hline Ear11 & 21.6494 & 55.383822 & 391.48428 & 977.02612 & 11.087313 & 13.730056 & 13.714121 \\
\hline Hdgfrp3 & 21.1601 & 1281.211 & 1298.9344 & 297.38562 & 25.220847 & 51.434399 & 40.268229 \\
\hline Colla2 & 20.5173 & 339.99871 & 4291.3582 & 5733.8908 & 83.98159 & 112.97263 & 102.09484 \\
\hline $\mathrm{Pbx} 3$ & 20.4553 & 676.6436 & 920.12073 & 266.60106 & 24.664333 & 30.784582 & 25.541661 \\
\hline Gm131 & 20.4465 & 398.62486 & 926.8408 & 163.44756 & 14.672889 & 25.276232 & 19.048693 \\
\hline Zfp503 & 19.7705 & 1001.7347 & 864.25303 & 1511.3059 & 47.633154 & 51.880398 & 68.514333 \\
\hline Cpsf41 & 19.4221 & 869.32006 & 1132.9344 & 546.39088 & 45.807816 & 40.828778 & 39.272828 \\
\hline Arg1 & 18.7917 & 153.49808 & 347.98141 & 362.23184 & 15.264449 & 12.73725 & 14.996629 \\
\hline Wdr25 & 18.7675 & 1022.6011 & 1617.1128 & 500.97945 & 45.55188 & 68.147554 & 40.373227 \\
\hline Hdgfrp3 & 17.3088 & 1801.4835 & 1881.5406 & 346.63431 & 55.433309 & 68.286603 & 59.855682 \\
\hline 2700008G24Rik & 16.8076 & 174.32627 & 279.9344 & 332.2112 & 12.826339 & 16.533927 & 16.100425 \\
\hline 1200009I06Rik & 16.7403 & 747.10833 & 712.62984 & 272.26834 & 24.462776 & 34.020859 & 37.128239 \\
\hline Heyl & 16.4924 & 883.69896 & 1176.2105 & 141.80943 & 37.03817 & 25.443133 & 34.867617 \\
\hline $\mathrm{Ccl} 8$ & 16.085 & 3122.5173 & 3681.4125 & 3081.2439 & 309.94325 & 151.60889 & 181.12498 \\
\hline Mum111 & 16.0618 & 111.56357 & 104.83175 & 182.2754 & 9.3458287 & 8.8661181 & 6.2088296 \\
\hline Mxra7 & 15.9612 & 859.21131 & 990.33206 & 598.63558 & 71.772885 & 48.45997 & 36.016372 \\
\hline Trim2 & 15.7122 & 390.82952 & 1081.5344 & 210.35548 & 26.216227 & 30.058828 & 29.08893 \\
\hline Trim2 & 14.7648 & 238.29582 & 592.27823 & 113.53876 & 13.654063 & 17.819311 & 20.46206 \\
\hline Sertad4 & 14.7645 & 518.53119 & 232.25101 & 43.401329 & 13.052917 & 11.92664 & 10.431711 \\
\hline
\end{tabular}




\begin{tabular}{|c|c|c|c|c|c|c|c|}
\hline Aldh $1 \mathrm{~b} 1$ & 14.1856 & 866.18017 & 3208.7547 & 471.99523 & 93.12193 & 71.945043 & 68.593929 \\
\hline Rab34 & 14.0889 & 353.89983 & 956.94962 & 391.92457 & 34.720094 & 32.674604 & 41.835974 \\
\hline Ifi202b & 13.6844 & 3678.8672 & 4728.4988 & 483.60013 & 124.10106 & 163.03114 & 162.25419 \\
\hline Dtx 1 & 13.1509 & 2517.9344 & 2405.1639 & 2169.4912 & 182.54685 & 181.70659 & 174.15517 \\
\hline Cxcl13 & 13.1001 & 839.87812 & 676.96211 & 199.98024 & 33.719741 & 42.929748 & 34.938255 \\
\hline Ptpn13 & 13.0557 & 694.38071 & 1020.0688 & 1228.6488 & 70.556257 & 77.832289 & 71.213209 \\
\hline Olfml3 & 12.6032 & 331.29315 & 770.59027 & 1131.5558 & 52.479539 & 43.849842 & 62.706652 \\
\hline Hes1 & 12.5858 & 1013.4099 & 1008.0545 & 533.63602 & 66.795563 & 56.60496 & 72.321398 \\
\hline Elav14 & 12.4072 & 405.70433 & 255.67526 & 15.155587 & 9.4512199 & 9.7444948 & 8.9372734 \\
\hline Sdc1 & 12.3095 & 611.15405 & 796.80583 & 540.45519 & 38.31298 & 63.521767 & 57.97954 \\
\hline Rab34 & 12.2142 & 221.69192 & 589.77297 & 285.99134 & 24.83712 & 23.456934 & 35.222449 \\
\hline LOC100041694 & 11.9083 & 542.84825 & 67.153281 & 241.81007 & 19.495749 & 14.949842 & 17.910085 \\
\hline Sdc1 & 11.8825 & 701.76773 & 1052.4412 & 651.39158 & 55.016542 & 73.265249 & 71.141462 \\
\hline Col3a1 & 11.6777 & 1041.3036 & 4849.4949 & 656.66643 & 77.934395 & 134.04424 & 199.33059 \\
\hline Prmt7 & 11.3792 & 2317.447 & 2871.1588 & 1922.235 & 213.36462 & 190.86626 & 213.1507 \\
\hline Slc $45 \mathrm{a} 3$ & 11.3543 & 179.97385 & 387.11276 & 314.68564 & 28.71702 & 21.828953 & 23.893202 \\
\hline H19 & 11.3335 & 24.075063 & 7995.143 & 61.680376 & 19.547898 & 19.616636 & 21.267967 \\
\hline $\operatorname{Tln} 2$ & 11.0018 & 463.76773 & 280.65413 & 137.07368 & 30.006227 & 17.280432 & 25.838437 \\
\hline Bmp7 & 10.7702 & 417.35106 & 532.08524 & 385.44253 & 39.101832 & 38.766376 & 45.19763 \\
\hline Treml2 & 0.0946 & 28.03108704 & 25.30750202 & 55.43439571 & 320.5967365 & 390.4343953 & 371.5278631 \\
\hline Tiparp & 0.0946 & 68.88878952 & 68.38389226 & 48.10728046 & 591.601062 & 668.7677287 & 677.0199327 \\
\hline Calcrl & 0.0917 & 15.63890907 & 36.86957835 & 129.7698851 & 355.0863099 & 433.752926 & 629.9343953 \\
\hline Hmgn3 & 0.0903 & 11.60994675 & 10.0788286 & 28.62748177 & 164.7406033 & 154.9269937 & 178.529699 \\
\hline Gpr174 & 0.088 & 13.42644495 & 13.8881514 & 23.02741987 & 195.4781102 & 176.4711499 & 182.8369489 \\
\hline St3gal6 & 0.0879 & 18.03867867 & 14.81986288 & 34.80290726 & 241.1592801 & 229.168287 & 247.8699584 \\
\hline Lypd6b & 0.0877 & 24.66290444 & 19.31466851 & 48.95239226 & 318.737146 & 367.378346 & 295.6010132 \\
\hline Bach2 & 0.0871 & 40.17932728 & 49.43440559 & 49.77801655 & 506.0822045 & 578.3769359 & 510.5529818 \\
\hline Tes & 0.0867 & 52.43439739 & 49.77613572 & 139.2660727 & 691.141747 & 857.9227717 & 941.4749662 \\
\hline Arap2 & 0.0861 & 29.37951067 & 19.9319749 & 57.99373182 & 326.7856653 & 394.8104734 & 412.2783797 \\
\hline Eif2s3y & 0.0858 & 473.5933247 & 9.871223988 & 24.33330108 & 638.2195894 & 513.601062 & 550.117434 \\
\hline Dnajc6 & 0.084 & 15.29866013 & 9.927639198 & 11.56575867 & 124.5560615 & 149.4931051 & 159.008556 \\
\hline Cxcr6 & 0.082 & 10.01080048 & 12.99371696 & 61.56649026 & 224.601062 & 247.4343953 & 261.7677287 \\
\hline Ly6d & 0.0812 & 53.56731234 & 47.58375353 & 189.7677287 & 1090.767729 & 919.716475 & 901.0770002 \\
\hline AW112010 & 0.0804 & 62.10106202 & 102.4343953 & 471.2677287 & 2123.82677 & 1820.12371 & 1489.767729 \\
\hline
\end{tabular}




\begin{tabular}{|c|c|c|c|c|c|c|c|}
\hline Dnajc6 & 0.0783 & 149.8855984 & 26.03693894 & 53.50285713 & 697.39574 & 747.7677287 & 834.7677287 \\
\hline Cc15 & 0.0782 & 61.4946868 & 78.12798995 & 208.9106998 & 1216.722583 & 1460.281593 & 1183.162003 \\
\hline Tctex $1 \mathrm{~d} 1$ & 0.0769 & 160.2677287 & 12.32157827 & 70.26772868 & 715.7249161 & 650.9993301 & 654.940627 \\
\hline Nebl & 0.0756 & 36.74082254 & 81.53343571 & 386.7757008 & 1246.204533 & 1401.509624 & 1537.954999 \\
\hline St3gal6 & 0.073 & 12.43755625 & 16.12016831 & 17.82809917 & 198.9914744 & 206.5852962 & 223.8733586 \\
\hline Ccnd2 & 0.0726 & 20.71626758 & 47.48686113 & 90.26772868 & 526.5371368 & 760.8521537 & 579.8028393 \\
\hline Cd69 & 0.0723 & 36.89573945 & 49.23013257 & 84.43439534 & 568.601062 & 767.601062 & 929.6854286 \\
\hline Ccnd2 & 0.0716 & 24.49741741 & 51.43439889 & 95.09769027 & 575.0537271 & 777.9529714 & 729.2721404 \\
\hline Tiparp & 0.0706 & 27.74032285 & 21.08894972 & 29.5194842 & 328.5988338 & 379.8822201 & 393.2067161 \\
\hline $\mathrm{I} 118 \mathrm{r} 1$ & 0.0668 & 24.79732127 & 29.76026207 & 88.50928385 & 501.9040287 & 731.5547929 & 596.9852836 \\
\hline 2610019F03Rik & 0.0668 & 36.03684742 & 21.20013148 & 158.9087977 & 707.4277305 & 748.0450242 & 767.9827369 \\
\hline Cend 2 & 0.0654 & 34.83934474 & 79.35021457 & 160.9363557 & 1079.988187 & 1218.993983 & 1208.845939 \\
\hline Ccnd 2 & 0.0644 & 49.11822147 & 124.6499699 & 240.5304103 & 1603.767729 & 1855.870715 & 1854.434395 \\
\hline 5830443L24Rik & 0.0636 & 88.34591359 & 28.0645787 & 264.2729139 & 1494.30804 & 1419.255947 & 1201.091476 \\
\hline Rag2 & 0.0631 & 63.7737682 & 153.0692755 & 19.08817773 & 789.7677287 & 835.9075374 & 1121.101062 \\
\hline Ccnd2 & 0.0589 & 18.15137414 & 31.57647413 & 79.76772868 & 514.1294179 & 632.6582276 & 687.101062 \\
\hline Cdc42ep3 & 0.0588 & 81.24821236 & 15.83749286 & 70.92441222 & 672.2355847 & 797.7057712 & 836.151814 \\
\hline Klrd1 & 0.0574 & 12.89620793 & 15.21227466 & 58.24413671 & 356.2071901 & 475.4787431 & 356.6735286 \\
\hline Armcx2 & 0.0513 & 13.75880825 & 12.54578745 & 74.31426863 & 479.6596755 & 414.2640287 & 478.7432834 \\
\hline LOC665506 & 0.0499 & 30.95068713 & 32.44299119 & 40.18983202 & 768.601062 & 688.4442198 & 613.7677287 \\
\hline Armcx 1 & 0.0486 & 14.08090185 & 17.66544267 & 19.70766874 & 341.1415191 & 343.2456355 & 364.3540873 \\
\hline Ccr9 & 0.0483 & 68.846513 & 119.1821634 & 991.3820388 & 4134.339253 & 3927.828341 & 4442.684395 \\
\hline Ccr9 & 0.0476 & 39.49142756 & 69.15583794 & 584.4230933 & 2701.529683 & 2154.290623 & 2540.860917 \\
\hline Ugt8a & 0.0459 & 6.159042801 & 275.8018179 & 6.930008352 & 412.269012 & 503.4036048 & 587.4760133 \\
\hline Gpr174 & 0.0455 & 10.02759314 & 17.06485037 & 43.99014487 & 372.0695058 & 458.3198546 & 469.3037382 \\
\hline Tcrb-V8.2 & 0.0438 & 19.04254901 & 15.22287772 & 990.2170438 & 1459.81797 & 1404.747226 & 1671.520596 \\
\hline S100a10 & 0.0396 & 42.03521032 & 61.32172159 & 128.3269606 & 1445.449919 & 1736.761028 & 2124.804773 \\
\hline S100a10 & 0.0394 & 59.81282621 & 77.43466166 & 127.2298994 & 1914.692382 & 2195.440276 & 2293.601062 \\
\hline 1700021K02Rik & 0.0341 & 12.47447791 & 16.71297155 & 34.61759624 & 437.8454094 & 581.1573895 & 713.3086516 \\
\hline Tcrb-V13 & 0.0264 & 19.95176963 & 12.36548994 & 21.70619148 & 701.7840597 & 676.9506898 & 611.1617623 \\
\hline 1700021K02Rik & 0.0222 & 5.042702665 & 6.658070982 & 32.77609728 & 310.3369626 & 502.500867 & 642.9040277 \\
\hline Tcrb-V13 & 0.0188 & 16.71766433 & 16.46766878 & 16.84377157 & 860.6042366 & 881.601062 & 925.9931538 \\
\hline
\end{tabular}

Red mark indicates up-regulated miRNAs in RTL tissues and Green mark indicates down-regulation. "C" represents control group that is normal normal thymus tissues; "T" represents RTL tissues. 
Tab S3. Statistic analysis of up-regulated miRNAs

\begin{tabular}{|c|c|c|c|c|c|}
\hline $\begin{array}{l}\text { Bonferroni's Multiple } \\
\text { Comparison Test }\end{array}$ & Mean Diff. & $\mathrm{t}$ & Significant? $\mathrm{P}<0.05$ ? & Summary & $95 \% \mathrm{Cl}$ of diff \\
\hline miR-762 vs miR-NC & 1105 & 8.521 & Yes & $* * *$ & 693.5 to 1516 \\
\hline miR-714 vs miR-NC & 1035 & 7.986 & Yes & 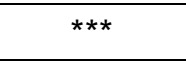 & 624.1 to 1447 \\
\hline miR-476a vs miR-NC & 1019 & 7.863 & Yes & $* * *$ & 608.1 to 1431 \\
\hline miR-455 vs miR-NC & 441.3 & 3.404 & Yes & $*$ & 30.12 to 852.5 \\
\hline miR-699 vs miR-NC & 1016 & 7.839 & Yes & 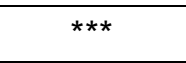 & 605.1 to 1428 \\
\hline miR-712 vs miR-NC & 686.0 & 5.291 & Yes & $* \star *$ & 274.8 to 1097 \\
\hline miR-685 vs miR-NC & 882.0 & 6.803 & Yes & $\star \star \star *$ & 470.8 to 1293 \\
\hline miR-705 vs miR-NC & 602.3 & 4.646 & Yes & $\star \star \star *$ & 191.1 to 1014 \\
\hline miR-494 vs miR-NC & 458.7 & 3.538 & Yes & ${ }^{*}$ & 47.46 to 869.9 \\
\hline miR-711 vs miR-NC & 426.7 & 3.291 & Yes & $*$ & 15.46 to 837.9 \\
\hline miR-181d vs miR-NC & 688.7 & 5.312 & Yes & $* * *$ & 277.5 to 1100 \\
\hline miR-149 vs miR-NC & 442.0 & 3.409 & Yes & $*$ & 30.79 to 853.2 \\
\hline miR-720 vs miR-NC & 415.7 & 3.206 & Yes & * & 4.458 to 826.9 \\
\hline miR-744 vs miR-NC & 465.0 & 3.587 & Yes & * & 53.79 to 876.2 \\
\hline miR-210 vs miR-NC & 435.3 & 3.358 & Yes & * & 24.12 to 846.5 \\
\hline
\end{tabular}


Tab S4. Statistic analysis of down-regulated miRNAs

\begin{tabular}{|l|l|l|l|l|l|}
\hline Bonferroni's Multiple Comparison Test & Mean Diff. & \multicolumn{1}{|c|}{$\mathrm{t}$} & Significant? $\mathrm{P}<0.05 ?$ & Summary & $95 \%$ Cl of diff \\
\hline miR-145 vs miR-NC & -67.33 & 8.748 & Yes & $* * *$ & -91.43 to -43.24 \\
\hline miR-376b vs miR-NC & -62.33 & 8.099 & Yes & $* * *$ & -86.43 to -38.24 \\
\hline miR-708 vs miR-NC & -70.67 & 9.181 & Yes & $* * *$ & -94.76 to -46.57 \\
\hline miR-152 vs miR-NC & -74.00 & 9.614 & Yes & $* * *$ & -98.09 to -49.91 \\
\hline miR-143 vs miR-NC & -80.67 & 10.48 & Yes & $* * *$ & -104.8 to -56.57 \\
\hline miR-200c vs miR-NC & -71.00 & 9.225 & Yes & $* * *$ & -95.09 to -46.91 \\
\hline miR-139b vs miR-NC & -66.00 & 8.575 & Yes & $* * *$ & -90.09 to -41.91 \\
\hline miR-203 vs miR-NC & -73.00 & 9.484 & Yes & $* * *$ & -97.09 to -48.91 \\
\hline miR-100 vs miR-NC & -73.00 & 9.484 & Yes & $* * *$ & -97.09 to -48.91 \\
\hline miR-99a vs miR-NC & -72.00 & 9.355 & Yes & $* * *$ & -96.09 to -47.91 \\
\hline miR-486 vs miR-NC & -85.00 & 11.04 & Yes & $* * *$ & -109.1 to -60.91 \\
\hline
\end{tabular}

